\title{
Atuação do enfermeiro na prevenção de lesão por pressão em paciente com COVID-
}

\section{9 na UTI}

Nurse's role in the prevention of pressure injuries in patients with COVID-19 in the ICU

Papel de la enfermera en la prevención de lesiones por presión en pacientes con COVID-19 en UCI

Rômulo Leno Miranda Barros ORCID: https://orcid.org/0000-0003-1802-4521

Universidade da Amazônia, Brasil

E-mail: romuloleno@hotmail.com Joelma de Sousa Araújo

ORCID: https://orcid.org/0000-0001-7043-6756

Universidade da Amazônia, Brasil

E-mail: joelmaaraujo2219@gmail.com

Antônio Andrey Padilha dos Reis

ORCID: https://orcid.org/0000-0001-8790-355X

Universidade da Amazônia, Brasil

E-mail: andreyreis3507@gmail.com

Rita do Socorro Ribeiro Quaresma Oliveira

ORCID: https://orcid.org/0000-0002-7397-6448

Universidade da Amazônia, Brasil E-mail: ritanhan@hotmail.com

Brenda Tanielle Dutra Barros

ORCID: https://orcid.org/0000-0002-3184-050X Centro Universitário Metropolitano da Amazônia, Brasil

E-mail: brendatanielle.enf@gmail.com

Virgínia Mercês Lara Pessoa Oliveira

ORCID: https://orcid.org/0000-0002-4417-637X

Centro Universitário Metropolitano da Amazônia, Brasil

E-mail: virginiaoliveira@famaz.edu.br

Rosana Cristina Coqueiro Campos

ORCID: https://orcid.org/0000-0003-2719-7248

Centro Universitário Metropolitano da Amazônia, Brasil

E-mail: rosanacampos06@yahoo.com.br

Mayra Gama Leão Pereira

ORCID: https://orcid.org/0000-0002-1050-4590 Universidade Federal do Pará, Brasil

E-mail: mayra_leao@live.com

Eugenia Mota Aguiar Milhomem

ORCID: https://orcid.org/0000-0002-7905-425X

Universidade da Amazônia, Brasil

E-mail: eugenia.aguiar@live.com

Daniel de Sarges Rodrigues

ORCID: https://orcid.org/0000-0001-9750-5280

Universidade da Amazônia, Brasil

E-mail: enfermeirodanrodrigues@gmail.com

Anderson de Jesus Franco Chagas

ORCID: https://orcid.org/0000-0002-0369-2327

Universidade da Amazônia, Brasil

E-mail: andersonchagas24@gmail.com

Silvani Damasceno de Barros

ORCID: https://orcid.org/0000-0002-4752-6312

Centro Universitário Metropolitano da Amazônia, Brasil

E-mail: silvanedamasceno123@gmail.com

Marcely Monteiro da Silva

ORCID: https://orcid.org/0000-0001-5878-5531

Centro Universitário Metropolitano da Amazônia, Brasil

E-mail: marcelym98@gmail.com

Taila Cristina Paiva da Costa

ORCID: https://orcid.org/0000-0003-0223-7813

Universidade da Amazônia, Brasil

E-mail: taylacosta-14@ hotmail.com 


\begin{abstract}
Resumo
Uma das consequências mais comuns resultante da permanência da hospitalização, em especial no setor de UTI, é o aparecimento de lesões na pele nos pacientes. Objetivos: analisar as evidências científicas disponíveis acerca atuação do enfermeiro no manejo do cuidado e na prevenção de lesões por pressões na UTI em paciente com COVID- 19 . Metodologia: De caráter exploratório, descritiva, com abordagem qualitativa, através de uma Revisão Integrativa de Literatura (RIL). A busca de dados foi realizada com base nos descritores: "Assistência à Saúde", "Infecções por Coronavirus", "Enfermagem"; "Lesão por pressão"; "Unidades de Terapia Intensiva", delimitando-se estudos publicados no recorte temporal entre 2019 a 2021. A coleta de dados foi realizada no período de agosto a outubro de 2021, nas bases de dados: LILACS, BDENF, SciELO, CAPES PUBMED e BIREME. Resultado e discussão: Apresentaram-se em três categorias temáticas: 1) impactos na atuação do enfermeiro na prevenção de lesão por pressão em paciente com COVID-19 na UTI; 2) o tempo de internação dos pacientes com covid-19 e a falta insumos; 3) os aspectos imunológicos do paciente internado na UTI com COVID-19. Observa-se que a LPP adquirida na UTI está associada ao aumento da morbidade e mortalidade, e é considerada como amplamente evitável. Conclusão: É responsabilidade dos profissionais de enfermagem realizar de forma primordial a avaliação dos fatores de riscos para o desencadeamento de LPP em seus pacientes, com a finalidade de prevenir e reconhece-las de maneira precoce, desenvolvendo parâmetros essenciais para a assistência ligada à integridade da pele do paciente.
\end{abstract}

Palavras-chave: Assistência à saúde; Infecções por Coronavírus; Enfermagem; Lesão por pressão; Unidades de terapia intensiva.

\begin{abstract}
One of the most common consequences resulting from hospitalization, especially in the ICU sector, is the appearance of skin lesions in patients. Objectives: to analyze the available scientific evidence about the role of nurses in the management of care and the prevention of pressure injuries in the ICU in patients with COVID-19. Methodology: Exploratory, descriptive, with a qualitative approach, through an Integrative Literature Review (RIL). The data search was performed based on the descriptors: "Health Care", "Coronavirus Infections", "Nursing"; "Pressure Injury"; "Intensive Care Units", delimiting studies published in the time frame between 2019 and 2021. Data collection was carried out from August to October 2021, in the databases: LILACS, BDENF, SciELO, CAPES PUBMED and BIREME. Results and discussion: They were presented in three thematic categories: 1) impacts on the role of nurses in the prevention of pressure injuries in patients with COVID-19 in the ICU; 2) the length of stay of patients with COVID-19 and lack of supplies and 3) the immunological aspects of the patient hospitalized in the ICU with COVID19. It is observed that PPL acquired in the ICU is associated with increased morbidity and mortality, and it is considered to be largely avoidable. Conclusion: It is the responsibility of nursing professionals to carry out primarily the assessment of risk factors for triggering PPL in their patients, in order to prevent and recognize them early, developing essential parameters for care linked to the integrity of the patient skin.
\end{abstract}

Keywords: Health care; Coronavirus infections; Nursing; Pressure injury; Intensive care unit.

\title{
Resumen
}

Una de las consecuencias más habituales derivadas de la hospitalización, especialmente en el sector de UCI, es la aparición de lesiones cutáneas en los pacientes. Objetivos: analizar la evidencia científica disponible sobre el papel del enfermero en el manejo del cuidado y la prevención de las lesiones por presión en la UCI en pacientes con COVID19. Metodología: Exploratoria, descriptiva, con abordaje cualitativo, mediante una Revisión Integrativa de la Literatura (RIL). La búsqueda de datos se realizó con base en los descriptores: "Atención de la salud", "Infecciones por coronavirus", "Enfermería"; "Lesión por presión"; "Unidades de Cuidados Intensivos", delimitando los estudios publicados en el marco temporal entre 2019 y 2021. La recolección de datos se realizó de agosto a octubre de 2021, en las bases de datos: LILACS, BDENF, SciELO, CAPES PUBMED y BIREME. Resultados y discusión: Fueron presentados en tres categorías temáticas: 1) impactos en el rol del enfermero en la prevención de lesiones por presión en pacientes con COVID-19 en UCI; 2) el tiempo de estancia de los pacientes con COVID-19 y la falta de insumos y 3) los aspectos inmunológicos del paciente hospitalizado en la UCI con COVID-19. Se observa que la LPP adquirida en la UCI se asocia a un aumento de la morbimortalidad, y se considera que es evitable en gran medida. Conclusión: Es responsabilidad de los profesionales de enfermería realizar principalmente la evaluación de los factores de riesgo para desencadenar LPP en sus pacientes, con el fin de prevenirlos y reconocerlos precozmente, desarrollando parámetros esenciales para el cuidado ligados a la integridad de la piel del paciente.

Palabras clave: Cuidado de la salud; Infecciones por coronavirus; Enfermería; Lesión por presión; Unidades de cuidados intensivos. 


\section{Introdução}

As Unidades de Terapia Intensiva (UTI) são caracterizadas por ser um local onde há um atendimento especializado de enfermagem aos pacientes de alta complexidade, o que potencializa a necessidade constante de um profissional qualificado para atuar nessa área. Isto porque, como é destinado a pacientes em estado crítico, há maior probabilidade de ocorrer Eventos Adversos (EA) (Otto et al., 2019).

Uma das consequências mais comuns resultante da permanência da hospitalização, em especial no setor de UTI é o aparecimento de lesões na pele que acometem pacientes hospitalizados, o que os tornam mais vulneráveis. Tais lesões na pele são chamadas lesões por pressão (LPP), um dano localizado na pele que resulta da pressão intensa e/ou prolongada em combinação com o cisalhamento (Vargas \& Santos, 2019).

A COVID-19 é uma doença respiratória infectocontagiosa causada pelo vírus SARSCoV2 e seus sintomas incluem: febre, dispneia e coriza. A forma mais grave é a Síndrome Respiratória Aguda Grave (SRAG), em que os indivíduos apresentam dispneia, saturação de oxigênio abaixo de 95\% em ar ambiente ou cianose em lábios ou face (Brasil, 2020).

Em torno de 10 a $15 \%$ dos pacientes irão evoluir para formas graves necessitando de suporte intensivo em Unidades de Terapia Intensiva (UTI). Para melhorar o padrão respiratório em pacientes com SRAG, uma das estratégias é o posicionamento em PRONA, em decúbito ventral com vistas a reduzir a compressão pulmonar e melhorar a perfusão. A posição PRONA pode levar ao desenvolvimento de lesões por pressão (LPP), sendo uma das principais complicações, devido a pressão exercida sobre as proeminências ósseas de ombros, cintura escapular, região frontal da cabeça, nariz e mandíbula. Além delas a posição PRONA aumenta o risco de edema facial, abrasões de córnea e extubação acidental (Pachá et al., 2020).

Alguns desafios circundam o manejo ao paciente no momento da prevenção de lesão por pressão no contexto do Coronavírus, pois existem as alterações resultantes da infecção provocadas pelo vírus que levam o paciente à instabilidade em seu quadro clínico como a diminuição da oxigenação nos tecidos, aumento do tempo de internação e dificuldades no reposicionamento deste, o culmina no aparecimento das LPs. Os fatores que envolvem recursos humanos e materiais também são decisivos para o controle das LPs, pois a escassez de ambos acaba limitando a equipe cuidadora, resultando em um cuidado com qualidade reduzida (Ramalho et al., 2020).

No Brasil os índices de incidência e prevalência são similares aos relatos na literatura mundial pelos números de casos novos de 38,9\% em pacientes internados desenvolvem algum tipo de lesão por pressão, por isso a importância em pesquisar o assunto e articular estratégias para diminui-las por meio da prevenção e da identificação de fatores de risco (Vargas \& Santos, 2019).

Para entender melhor a problemática é necessário conhecer sua incidência e prevalência; ressalta-se a importância da temática do presente estudo, contribuindo para mais discussão do tema na comunidade cientifica, seja na prevenção ou no tratamento da LPP. Sua incidência é considerada um importante indicador da qualidade assistencial de enfermagem, pois permite pesquisar os casos de acordo com sua distribuição (Pachá et al., 2020).

O estudo justifica-se pela necessidade de conhecer sobre a atuação do enfermeiro no manejo do cuidado e na prevenção das LPPs dos pacientes com COVID-19 internados na UTI, objetivando melhor compreensão de como esse evento ocorre e impacta o cotidiano da equipe. Destaca-se que a enfermagem se apresenta como uma ciência que tem como característica o cuidado humanizado. Neste sentido, o aspecto preventivo, bem como o de promoção da saúde, busca nortear a prática assistencial para minimizar os índices de lesão por pressão.

Diante disso, surge a pergunta problema de pesquisa: como é atuação do enfermeiro no manejo do cuidado e na prevenção de lesão por pressão em pacientes com COVID-19 na UTI, evidenciado na literatura científica dos últimos dois anos?

O estudo objetivou conhecer e revelar as evidências cientificas disponíveis acerca da atuação do enfermeiro no 
manejo do cuidado e na prevenção de lesões por pressões na UTI em paciente com COVID-19.

\section{Metodologia}

A presente pesquisa baseia-se em um estudo do tipo revisão integrativa (RI) de literatura com abordagem qualitativa, desenvolvido no período de junho a outubro de 2021, seguindo as seis fases do processo de elaboração de Revisão Integrativa de Literatura: $1^{\circ}$ Etapa - identificação do tema e seleção da hipótese ou questão de pesquisa para a elaboração da revisão integrativa. $2^{\circ}$ Etapa - Procurar a melhor evidência. $3^{\circ}$ Etapa - Avaliar criticamente as evidências. $4^{\circ}$ Etapa - Integrar as evidências. $5^{\circ}$ Etapa - discussão dos resultados. $6^{\circ}$ Etapa - apresentação da síntese do conhecimento produzido; revisão/síntese do conhecimento. (Teixeira et al., 2014; Botelho, Cunha \& Macedo, 2011.)

Na primeira etapa elaborou-se a seguinte questão de pesquisa: "Qual é atuação do enfermeiro no manejo do cuidado e na prevenção de LPP em pacientes com COVID-19 na UTI, evidenciados na literatura científica de 2019 a 2021 ?".

$2^{\circ}$ Etapa Busca na literatura: fase que correspondeu à busca variada e abrangente dos artigos em base de dados Literatura Latino-Americana e do Caribe em Ciências da Saúde (LILACS), Base de Dados de Enfermagem (BDENF), Scientifc Electronic Library Online (SciELO), banco de teses (CAPES) PUBMED, BIREME, biblioteca virtual - MS, acessada pelo site da biblioteca virtual em saúde (BVS).

Foram incluídos artigos científicos disponíveis nas bases de dados estipuladas, cuja busca foi realizada por meio dos descritores em Ciências da Saúde (DeCS): "Assistência à Saúde", "Infecções por Coronavirus", "Enfermagem”, "Lesão por pressão" e "Unidades de Terapia Intensiva", Os mesmos foram cruzados entre si, a partir da utilização do operador booleano “AND” na busca por produções, publicados na íntegra, nacionais, no período de 2019 a 2021, nacionais e internacionais, gratuitos, estudos na integra e primários e para evidenciar este processo foi utilizado o Fluxograma PRISMA a seguir: 
Fluxograma 1: Fluxograma Prisma de identificação e síntese das buscas e resultados da pesquisa.

\section{FLUXOGRAMA- PRISMA}
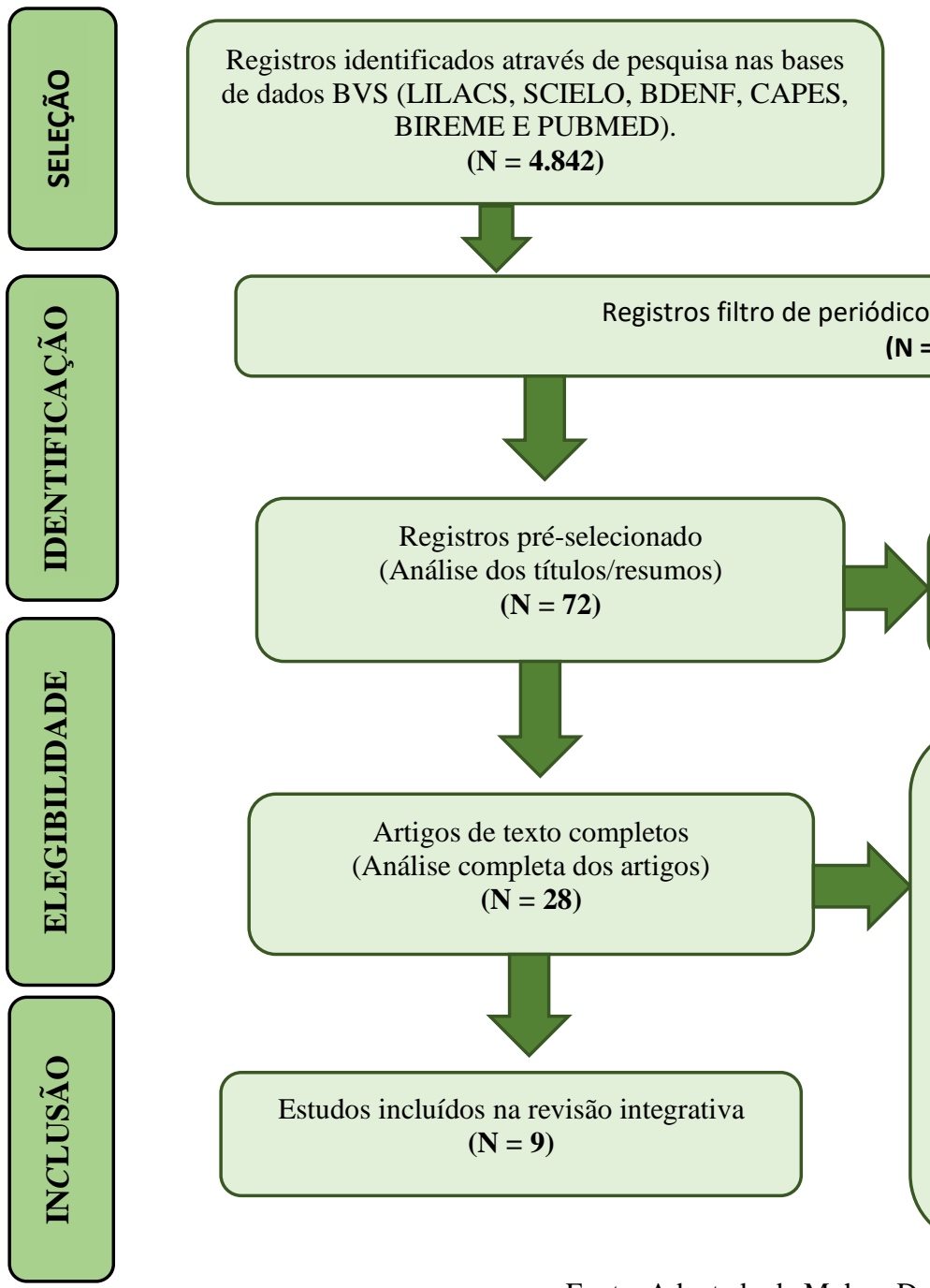

Registros adicionados por meio de outros critérios/métodos (livros e jornais) $(\mathbf{N}=\mathbf{0 0})$ (ED).

$(\mathrm{n}=0 \mathrm{n})$

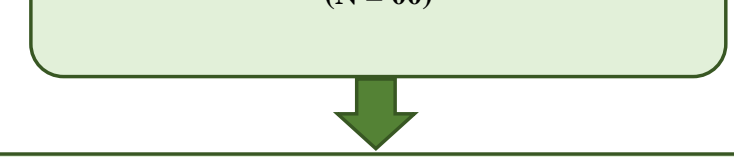

$(N=2.217)$

Artigos de texto completos excluídos $(\mathbf{N}=\mathbf{1 9})$, sendo:

Registros excluídos (Falta de enquadramento) $(\mathbf{N}=\mathbf{4 4})$

09 Estão duplicados nas bases de dados.

02 Trabalhos de conclusão de curso,

08 Artigos secundários, revisões integrativas.

Fonte: Adaptado de Moher, D., et al. (2009).

Na terceira etapa foi à fase de recolhimento dos artigos previamente selecionados e coletados as informações por meio da utilização de um instrumento de URSI, com a finalidade de minimizar o risco de erros na transcrição, assegurar precisão na checagem das informações e utilizar como registro.

Para extrair os dados dos artigos selecionados utilizou-se o formulário de Ursi (2005) incluindo: definição dos sujeitos, metodologia, tamanho da amostra, mensuração de variáveis, método de análise e conceitos bases empregadas (Soares et al., 2014)

$\mathrm{Na}$ quarta etapa organizou-se a integração das evidências dos artigos selecionados, para tal elaborou-se quadros sínteses que possibilitou a análise das informações e categorização dos conteúdos analisados. Uma matriz de síntese pode conter informações verbais, conotações, resumos de texto, extratos de notas, memorandos, respostas padronizadas e, em geral, dispor de dados integrados em torno de um ponto ou temas de pesquisa (Botelho, Cunha \& Macedo, 2011). O objetivo do instrumento foi verificar a percepção dos enfermeiros sobre o que considera como ações de prevenções de LPP, seu conhecimento sobre o manejo e o cuidado dessas lesões em pacientes com COVID-19 e sua a atuação nesses casos. 
A análise dos resultados foi realizada pela metodologia de Bardin. No que diz respeito à metodologia de Bardin, tal método se se resume a um conjunto de técnicas de análise das comunicações, visando obter, por procedimentos sistemáticos e objetivos de descrição e indicadores que permitam a inferência de conhecimentos relativos às condições de produção e recepção de mensagens. (Bardin, 2012; Urquiza, 2016).

Para cumprir a quinta e sexta etapas da revisão os resultados foram interpretados e discutidos, finalizando com a apresentação da revisão com abordagem qualitativa. Finalizando com a apresentação da revisão com abordagem qualitativa. A partir da análise dos quadros sínteses identificaram-se três categorias temáticas.

\section{Resultados}

A síntese dos resultados da pesquisa foi comparada ao referencial teórico, onde foi possível identificar as evidências sobre o objeto de estudo. Foi realizada uma pesquisa nas bases de dados por meio do cruzamento dos descritores definidos no item 3.3.1.

Desta pesquisa foram selecionados 09 (nove) artigos pelos critérios de inclusão e exclusão, em meio a 4.842 artigos encontrados em dados de cada artigo, de acordo com o formulário de Ursi (2005) adaptado e obtiveram os resultados de acordo com os descritores com os cruzamentos “AND" e de acordo com as bases de dados, expostos nas Tabelas 1 e 2, a seguir:

Tabela 1 - Distribuição dos artigos encontrados de acordo com os descritores com os cruzamentos AND nas bases de dados.

\begin{tabular}{|c|c|c|c|c|c|c|}
\hline \multirow[t]{2}{*}{ DESCRITORES } & \multicolumn{6}{|c|}{ BASE DE DADOS } \\
\hline & LILACS & SCIELO & BDENF & CAPES & PUBMED & BIREME \\
\hline Lesão por pressão $A N D$ Enfermagem & 77 & 1009 & 92 & 112 & 176 & 231 \\
\hline Infecções por Coronavirus $A N D$ Lesão por pressão & 5 & 111 & 4 & 71 & 28 & 41 \\
\hline TOTAL & \multicolumn{6}{|c|}{4.842} \\
\hline
\end{tabular}

Fonte: Autores.

Dos 4.842 artigos achados, foram excluídos: 2.217 fora do periódico nas bases de dados, 2.553 após Análise dos títulos, 44 resumos (Falta de enquadramento), 09 duplicados nas bases de dados, 02 Trabalhos de conclusão de curso, 08 Artigos de revisão integrativa. Restando assim 9 artigos selecionados para a revisão integrativa de literatura, conforme descreve o Fluxograma 1 de amostragem. 
Tabela 2 - Distribuição dos artigos selecionados de acordo com as bases de dados.

\begin{tabular}{|c|c|c|}
\hline \multirow[t]{2}{*}{ BASE DE DADOS } & \multicolumn{2}{|c|}{ ARTIGOS } \\
\hline & $\mathbf{N}^{\circ}$ & $\%$ \\
\hline LILACS & 2 & $22 \%$ \\
\hline SCIELO & 3 & $33 \%$ \\
\hline BDENF & 1 & $11 \%$ \\
\hline CAPES & 1 & $11 \%$ \\
\hline PUBMED & 2 & $22 \%$ \\
\hline BIREME & 0 & $0 \%$ \\
\hline TOTAL & 09 & $100 \%$ \\
\hline
\end{tabular}

Fonte: Autores.

De acordo com os dados da Tabela 2 verifica-se que 09 artigos foram encontrados, sendo que: 2 foram selecionados na base de dados LILACS, 3 no SCIELO, 1 na BVS/BDENF, 1 no CAPES e no PUBMED 2.

A distribuição dos artigos incluídos no estudo foram realizadas de acordo com o periódico, os autores, as bases de dados e com ano de publicação, como pode ser observado no Quadro 1, a seguir:

Quadro 1 - Síntese: Caracterização dos artigos.

\begin{tabular}{|c|c|c|c|c|}
\hline COD & TÍTULO & $\begin{array}{l}\text { PERIÓDICO } \\
\text { CIENTÍFICO }\end{array}$ & $\begin{array}{l}\text { BASE DE } \\
\text { DADOS }\end{array}$ & ANO \\
\hline A01 & $\begin{array}{l}\text { Lesão por pressão relacionada ao uso de equipamentos de } \\
\text { proteção individual na pandemia da COVID-19 }\end{array}$ & REBEN & BIREME & 2020 \\
\hline A02 & $\begin{array}{l}\text { Fatores de risco para o desenvolvimento de lesão por pressão } \\
\text { em pacientes críticos }\end{array}$ & Enfermagem Foco & SCIELO & 2019 \\
\hline A03 & $\begin{array}{l}\text { Reflexões sobre as recomendações para prevenção de lesões } \\
\text { por pressão durante a pandemia de COVID-19 }\end{array}$ & ESTIMA Braz. J & LILACS & 2020 \\
\hline A04 & $\begin{array}{l}\text { Lesão por Pressão em Unidade de Terapia Intensiva: estudo } \\
\text { de caso-controle }\end{array}$ & REBEN & SCIELO & 2020 \\
\hline A05 & $\begin{array}{l}\text { Posição PRONA como ferramenta emergente na assistência } \\
\text { ao paciente acometido por COVID-19: scoping review }\end{array}$ & $\begin{array}{l}\text { Rev. Latino } \\
\text { Enfermagem. }\end{array}$ & SCIELO & 2021 \\
\hline A06 & $\begin{array}{l}\text { Acute skin failure e lesão por pressão em paciente com } \\
\text { COVID-19 }\end{array}$ & ESTIMA Braz. J & BDENF & 2020 \\
\hline A07 & $\begin{array}{l}\text { Zero incidência de lesão por pressão em uma Unidade de } \\
\text { terapia intensiva covid-19 e o Fundamental envolvimento do } \\
\text { enfermeiro }\end{array}$ & $\begin{array}{l}\text { Centro de ensino e } \\
\text { pesquisa }\end{array}$ & CAPES & 2021 \\
\hline A08 & $\begin{array}{l}\text { COVID-19 patients in prone position: validation of } \\
\text { instructional materials for pressure injury prevention }\end{array}$ & REBEN & PUBMED & 2020 \\
\hline A09 & $\begin{array}{l}\text { Facial pressure ulcers in COVID-19 patients undergoing } \\
\text { prone positioning: How to prevent an underestimated } \\
\text { epidemic? }\end{array}$ & Elsevier Masson & PUBMED & 2020 \\
\hline
\end{tabular}

Fonte: Autores. 
Para demonstrar a síntese das contribuições dos estudos selecionados, utilizou-se um quadro (Quadro 2) contendo a distribuição dos artigos incluídos de acordo com o objetivo, metodologia e os achados da pesquisa.

Quadro 2 - Síntese: Contribuições dos estudos.

\begin{tabular}{|c|c|c|c|}
\hline CÓD & OBJETIVO & METODOLOGIA & RESULTADOS \\
\hline A01 & $\begin{array}{l}\text { Descrever prevalência e } \\
\text { fatores associados da lesão } \\
\text { por pressão relacionada ao } \\
\text { uso de equipamentos de } \\
\text { proteção individual durante a } \\
\text { pandemia da COVID-19. }\end{array}$ & $\begin{array}{l}\text { Estudo transversal realizado por meio de } \\
\text { instrumento disponibilizado em redes sociais } \\
\text { com } 1.106 \text { profissionais de saúde. Os dados } \\
\text { foram analisados por meio de estatística } \\
\text { descritiva. }\end{array}$ & $\begin{array}{l}\text { Houve prevalência de } 69,4 \% \text { para lesão por pressão } \\
\text { relacionada ao uso do equipamento de proteção individual, } \\
\text { com uma média de } 2,4 \text { lesões por profissional. Os fatores } \\
\text { significativos foram: menores de } 35 \text { anos de idade, trabalhar e } \\
\text { fazer uso de equipamentos de proteção individual por mais de } \\
\text { seis horas no dia, em unidades hospitalares e sem o uso de } \\
\text { insumos para proteção. }\end{array}$ \\
\hline A03 & $\begin{array}{l}\text { Refletir sobre } r \text { as } \\
\text { recomendações para prevenir } \\
\text { LPPs em pacientes afetado } \\
\text { pela COVID-19 em terapia } \\
\text { intensiva }\end{array}$ & $\begin{array}{l}\text { Estudo do tipo reflexão } \text { teórica } \\
\text { fundamentada no conceito de LPP definido } \\
\text { pelo National Pressure Injury Advisory } \\
\text { Panel }\end{array}$ & $\begin{array}{l}\text { As informações foram postas em três eixos temáticos: fatores } \\
\text { de risco de desenvolvimento de LPP específica para o paciente } \\
\text { com COVID-19; recomendações internacionais sobre LPP em } \\
\text { pacientes com COVID-19 e desafios para implementação das } \\
\text { recomendações de prevenção de LPP frente à pandemia de } \\
\text { COVID-19 no cenário brasileiro. }\end{array}$ \\
\hline A04 & $\begin{array}{l}\text { Avaliar a relação entre a } \\
\text { presença/ausência de Lesão } \\
\text { por Pressão e fatores } \\
\text { sociodemográficos e da } \\
\text { internação. }\end{array}$ & $\begin{array}{l}\text { Estudo de caso-controle, realizado por meio } \\
\text { de regressão logística múltipla com base em } \\
\text { informações dos sistemas de notifi cação de } \\
\text { eventos adversos e do sistema de informação } \\
\text { hospitalar. }\end{array}$ & $\begin{array}{l}\text { Entre os fatores de risco, destacaram-se, após ajuste, idade } \\
\text { maior ou igual } 60 \text { anos, internação por doenças infecciosas, } \\
\text { parasitárias e neoplasias, períodos de internação maiores que } \\
\text { sete dias e estar internado em UTI que não fosse UTI } \\
\text { convênio. A maioria das lesões foi notifi cada com grau de } \\
\text { dano leve e classifi cada em estágio II. }\end{array}$ \\
\hline A05 & $\begin{array}{l}\text { Descrever as evidências } \\
\text { científicas acerca da } \\
\text { utilização da posição prona na } \\
\text { assistência ao paciente com } \\
\text { insuficiência respiratória } \\
\text { aguda provocada por COVID- } \\
19 .\end{array}$ & $\begin{array}{l}\text { Trata-se de uma scoping review. O } \\
\text { instrumento PRISMA Extension for Scoping } \\
\text { Reviews foi utilizado para a redação do } \\
\text { estudo. As buscas foram realizadas em sete } \\
\text { bases de dados, resultando em } 2.441 \text { estudos } \\
\text { dos quais } 12 \text { compõem. a amostra. Uma } \\
\text { análise descritiva dos dados foi realizada } \\
\text { empregando frequências relativas e } \\
\text { absolutas. }\end{array}$ & $\begin{array}{l}\text { A utilização da posição prona ocorreu principalmente em } \\
\text { Unidades de Terapia Intensiva, com duração mínima de } 12 \text { a } \\
16 \text { horas, e teve como fundamentos de indicação critérios } \\
\text { específicos, tais como a relação } \mathrm{PaO} 2 / \mathrm{FiO} 2 \text {, a saturação de } \\
\text { oxigênio e a frequência respiratória. As complicações mais } \\
\text { prevalentes da sua utilização foram: extubação acidental, lesão } \\
\text { por pressão e edema facial. Identificou-se a redução da } \\
\text { hipoxemia e da mortalidade como principais desfechos } \\
\text { evidenciados na amostra. }\end{array}$ \\
\hline A06 & $\begin{array}{l}\text { Relatar o caso de um paciente } \\
\text { crítico com COVID-19 e } \\
\text { mostrar os principais achados } \\
\text { relacionados à lesão } \\
\text { considerada Acute skin failure } \\
\text { (ASF), bem como realizar seu } \\
\text { diagnóstico diferencial com } \\
\text { lesão por pressão } \quad \text { (LP) } \\
\text { evitável. }\end{array}$ & $\begin{array}{l}\text { Estudo observacional do tipo relato de caso, } \\
\text { desenvolvido em um hospital de São Paulo, } \\
\text { na unidade de terapia intensiva (UTI) } \\
\text { exclusiva a pessoas diagnosticadas com } \\
\text { COVID-19. Os dados foram coletados de um } \\
\text { único paciente, entre os meses de março e } \\
\text { setembro de } 2020 \text {. }\end{array}$ & $\begin{array}{l}\text { Paciente com complicações da COVID-19 evoluiu com lesão } \\
\text { de pele, inicialmente definida como LP e posteriormente } \\
\text { reclassificada como ASF. Os seguintes achados corroboraram } \\
\text { o diagnóstico: ventilação mecânica invasiva prolongada, } \\
\text { insuficiências respiratória, renal e cardíaca e sepse de foco } \\
\text { respiratório. Além disso, outros fatores agravantes, como o } \\
\text { uso de droga vasoativa, instabilidade hemodinâmica com } \\
\text { intolerância ao mínimo reposicionamento, jejum prolongado e } \\
\text { coagulopatia intravascular disseminada associada à infecção } \\
\text { pelo coronavírus. }\end{array}$ \\
\hline A07 & $\begin{array}{l}\text { Destaca-se a equipe de } \\
\text { enfermagem } \\
\text { protagonista na prevenção de } \\
\text { LPP's e precursora da } \\
\text { adequação de novas rotinas e } \\
\text { protocolos. }\end{array}$ & $\begin{array}{l}\text { Estudo do tipo relato de caso, que ocorreu } \\
\text { em hospital de grande porte em Aracaju-SE, } \\
\text { em uma unidade de terapia intensiva para } \\
\text { atendimento exclusivo de COVID-19. }\end{array}$ & $\begin{array}{l}\text { A partir das ações instituídas, observou-se queda na densidade } \\
\text { de incidência de lesão por pressão ressaltando densidade zero } \\
\text { em quatro meses no segundo semestre de } 2020\end{array}$ \\
\hline A08 & $\begin{array}{l}\text { Realizar a validação de } \\
\text { conteúdo e face de uma lista } \\
\text { de verificação e um banner } \\
\text { sobre prevenção de lesões por } \\
\text { pressão em pacientes em } \\
\text { decúbito ventral }\end{array}$ & $\begin{array}{l}\text { Trata-se de um estudo metodológico de } \\
\text { validação de conteúdo e face com } 26 \\
\text { enfermeiros com especialização. Os } \\
\text { profissionais avaliaram o checklist e o } \\
\text { banner em relação à clareza, relevância } \\
\text { teórica, relevância prática, relação das } \\
\text { figuras com o texto e tamanho da fonte }\end{array}$ & $\begin{array}{l}\text { Todas as ações descritas no checklist e no banner tiveram } \\
\text { Índice de Validade de Conteúdo superior a } 0,80 \text {, com } \\
\text { padronização do tempo verbal e ajustes estéticos no layout do } \\
\text { banner, conforme sugerido. }\end{array}$ \\
\hline
\end{tabular}




\begin{tabular}{|c|l|l|l|}
\hline \multirow{8}{*}{ A09 } & $\begin{array}{l}\text { Foi descrito dois casos com o } \\
\text { objetivo de enfatizar o risco } \\
\text { de úlcera por pressão facial } \\
\text { em decorrência da posição } \\
\text { prona, a fim de discutir sua } \\
\text { fisiopatologia e destacar a } \\
\text { importância de medidas } \\
\text { preventivas adequadas. }\end{array}$ & $\begin{array}{l}\text { Procuramos descrever a apresentação clínica } \\
\text { enfatizar o risco de úlcera por pressão de } \\
\text { face em decúbito ventral, discutir sua } \\
\text { fisiopatologia e destacar a importância de } \\
\text { ações preventivas adequadas. }\end{array}$ & $\begin{array}{l}\text { A posconama terapia adjuvante usada para tratar a } \\
\text { pneumonia por COVID-19 complicada por síndrome do } \\
\text { prolongada na pele facial ao nível das estruturas ósseas pode } \\
\text { ser responsável pelas úlceras de pressão facial. No contexto de } \\
\text { pneumonia COVID-19 grave, formulamos a hipótese de que a } \\
\text { hipoxemia, lesão microvascular e trombose podem aumentar o } \\
\text { risco de úlceras de pressão. }\end{array}$ \\
\hline
\end{tabular}

Fonte: Autores.

\subsection{Categorização e análise dos estudos selecionados}

A partir da análise dos quadros sínteses identificaram-se três categorias temáticas:

\subsubsection{Categoria 1: Impactos na atuação do enfermeiro na prevenção de lesão por pressão em paciente com COVID-19 na} $\boldsymbol{U T I}$

Nesta categoria, identificaram-se quatro artigos que tiveram como objetivo identificar, caracterizar a prevenção de LP no contexto do coronavírus, que se apresenta como uma situação ainda mais desafiadora, pois as alterações decorrentes da infecção expõem o paciente à maior instabilidade, menor oxigenação tecidual, tempo de internação em unidade crítico prolongado e possível dificuldade de reposicionamento, fatores que favorecem o desenvolvimento das LPs. Além disso, associam-se aos aspectos relacionados aos serviços de saúde, tais como escassez de materiais e tecnologias para prevenção, recursos humanos limitados, entre outros (Coelho et al., 2020).

Para prevenção destes incidentes, algumas estratégias devem ser aplicadas em todo paciente institucionalizado. A inspeção da pele na admissão de todo cliente, pois ao avalia-la verifica-se a integridade bem como a existência de LPP já instalada. Empregar na rotina a Escala de Braden ou qualquer outra escala que mensure e avalie os fatores de risco individuais de cada paciente. Supervisionar o paciente, condicionando-o seco e com a pele hidratada. Garantir nutrição adequada e hidratação. Dirimir a pressão, redistribuindo-a sobre demais proeminências ósseas (Machado et al., 2019).

Os estudos mostraram que a maioria das dificuldades apontadas pelos profissionais da equipe de enfermagem na implementação das etapas essenciais na prevenção de LPP, observou-se que alguns pontos foram relatados como problemas na realização de todas as etapas essenciais, desde a admissão do paciente, dentre eles, estão: a superlotação de pacientes, a falta de recursos materiais, a falta de conhecimento das etapas essenciais para prevenção de LPP e a falta de capacitação continuada sobre esta temática não são vistos como problemas para implementação desta conduta, desde o momento em que o paciente é admitido no serviço de saúde (Coelho et al., 2020).

Sobre isso, observou-se no estudo Ramalho et al., 2020 que a prevenção de LPP na posição PRONA é um desafio para a equipe multidisciplinar. Os pacientes em cuidados intensivos podem apresentar deterioração da integridade da derme em questão de horas. Além dos pontos de pressão natural no corpo decorrente do decúbito proposto, outro fator agravante são os múltiplos dispositivos médicos utilizados na terapia intensiva. Dentre esses, temos: o tubo orotraqueal, cateteres, drenos e sonda. As intervenções mais relevantes para prevenção de LPP no paciente submetido a decúbito ventral são:

- Escolha adequada da superfície de suporte, de forma que promova a redistribuição de pressão, além de fazer uso de dispositivos auxiliares para o posicionamento, tais como coxins e travesseiros para alívio de pressão;

- Inspeção rigorosa da pele antes da realização da PRONA, com intuito de proteger as áreas que apresentam maior risco de lesionar, sendo indicada a cobertura profilática em proeminências ósseas. Cita-se os principais pontos de pressão nesse posicionamento: testa bochecha, queixo, clavícula, cotovelos, genitais, pelve, joelhos, dorso e dedos dos pés e ao redor dos dispositivos de acesso. 
- Higienização cutânea adequada, com produtos adequados. Priorização das áreas corporais onde a umidade oferece risco maior para LP, como exemplo, pacientes com incontinência urinária, fecal e/ou mista.

- Reposicionamentos suscintos do paciente a cada duas ou quatro horas, com a utilização da técnica de posição nadador, onde se alterna a posição dos membros superiores e da cabeça. A depender das condições de trabalho e do quantitativo de pessoal disponível para o número de pacientes internados, pode haver sobrecarrega de profissionais, tornando dificultosa a execução do cuidado e as mudanças de decúbito preconizadas (Ramalho et al., 2020).

\subsubsection{Categoria 2: $O$ tempo de internação dos pacientes com covid-19 e a falta insumos}

Dos estudos pesquisados três abordaram as características dos pacientes que desenvolvem as LPPs, destacam assim que; a internação prolongada é um fator de risco importante. Sua ocorrência eleva a possibilidade de desenvolvimento de LPP. Entretanto os medicamentos de uso contínuo também podem contribuir para o aparecimento de LPP. Fármacos sedativos prejudicam a mobilização do sujeito e anti-hipertensivos diminuem o fluxo sanguíneo e a perfusão tecidual, o que eleva a predisposição do indivíduo às LPP (Machado et al., 2019).

Outro aspecto que se chama atenção é que pacientes intubados, em regime de ventilação mecânica, têm maior risco de apresentar LPP, que são resultantes das condições de instabilidade clínica do paciente, como restrição ao leito, percepção dos sentidos prejudicadas devido às suas condições clínicas e hemodinâmicas comprometidas, entre outros fatores. imobilidade no leito, percepção sensorial diminuída, entre outros fatores (Ramalho et al., 2020).

A complexidade e a gravidade dos pacientes internados resultam na necessidade de reavaliação diária do potencial e do risco de desenvolvimento de LPP. A reavaliação diária permite aos profissionais de saúde ajustar sua estratégia de prevenção conforme as necessidades do paciente. O grau de risco, conforme especificado em várias ferramentas, permite que os profissionais implantem estratégias individualizadas para os pacientes. Essa avaliação deve levar em consideração as fragilidades, vulnerabilidades e fatores de risco para o desenvolvimento de alterações de pele (Brasil, 2017).

Tendo em vista todos esses cuidados que visam à prevenção de LPP, a preocupação da equipe aumenta quando nos referimos a pacientes internados em UTI, que, na maioria das vezes, encontra-se em sedo-analgesia, restritos ao leito, em uso de múltiplos dispositivos e com alterações fisiológicas severas, fatores esses que favorecem o surgimento de LPP. Vários fatores de risco estão associados com o desenvolvimento de LPP em pacientes críticos, tais como: alterações do nível de consciência, déficit nutricional, pressão extrínseca associada à idade avançada, umidade, imobilidade no leito, período prolongado de internação, perfusão tecidual diminuída, uso de drogas vasoativas, sepse, sedação e as comorbidades como diabetes mellitus e doença vascular (Otto et al., 2019)

\subsubsection{Categoria 3: Os aspectos imunológicos do paciente internado na UTI com COVID-19}

Dos nove estudos pesquisados, quatro abordaram sobre o perfil epidemiológico dos pacientes com COVID-19, assim nesta categoria serão abordadas características e os aspectos imunológicos do paciente internado na UTI com COVID-19.

Foi observado na literatura que durante a internação na UTI, existem situações que comprometem a perfusão tissular da pele, como: redução da pressão sanguínea decorrente de alterações cardiovasculares, síndrome da resposta inflamatória sistêmica (SIRS), choque séptico, choque hemorrágico, uso de fármacos e instabilidade hemodinâmica. Em qualquer uma dessas situações, o paciente crítico sofre com as alterações do fluxo sanguíneo para a área que está sobre pressão, comprometendo a oxigenação e a nutrição dos tecidos naquele naquela região, podendo levar ao desenvolvimento de isquemia, hipóxia, edema e necrose tecidual (Otto et al., 2019)

Acredita-se que os principais mecanismos responsáveis pela gravidade nesses pacientes estão relacionados com a liberação exacerbada de mediadores inflamatórios e a desregulação do sistema de coagulação, o que favorece o 
desenvolvimento da SRAG e predispõe à necessidade de suporte respiratório, além da instabilidade clínica e hemodinâmica (Ramalho et al., 2021).

Um estudo apontou que, mesmo que as lesões apresentem-se com áreas cianóticas, não se deve confundi-las com as LPs tissulares profundas. É sempre importante realizar a avaliação da área que foi sujeitada a pressão e cisalhamento. O favorecimento da coagulopatia sistêmica resultante da infecção pelo Sars-CoV-2, está diretamente relacionada às diversas manifestações cutâneas no paciente, visto que a coagulopatia é desencadeada pela hipercoagulação microvascular. (Ramalho et al., 2020).

Quanto às variáveis clínicas dos pacientes com LPP, é possível observar que o gênero masculino é predominante. Predomínio da cor de pele branca e a média de idade dos pacientes com LPP foi menor. Quanto maior o tempo de internação, maior o risco de LPP. Desta forma, é possível considerar que os pacientes submetidos a um período prologado de uso de ventilação mecânica, sedação contínua, dias de balanço hídrico positivo e uso de antibióticos estão mais suscetíveis a desenvolver LPP (Coelho et al., 2020).

Em relação as questões nutricionais, o paciente que apresenta deficiência neste aspecto de sua saúde, o chamado Estado Hipercatabólico, com sua causística associada à COVID-19, é comum o desencadeamento de diarreia e por consequência, a elevação da umidade cutânea e dermatites relacionadas à incontinência (Ramalho et al., 2020).

\section{Discussão}

Observa-se na categoria 1 que a LPP adquirida na UTI em pacientes com COVID-19, está associada ao aumento da morbidade e mortalidade, e é considerada como amplamente evitável. Sua prevalência é considerada um marcador da qualidade dos cuidados de saúde. É imprescindível que o enfermeiro realize um controle rigoroso dos dias de internação do paciente em UTI. Assim que ele estiver estabilizado hemodinamicamente, deve ser realizada a mudança de decúbito e a retirada do leito o mais precoce possível (Pacha et al., 2018).

Sobre isso, as práticas das atribuições próprias do enfermeiro são protegidas pelo COFEN, de acordo com a Resolução COFEN n n 567 de 2018, o profissional de enfermagem tem apoio técnico para avaliação, classificação e tratamento das lesões cutâneas nos diferentes níveis de assistência à saúde, capacidade para atuar como vigilante na prevenção e no tratamento dessas lesões, atuar no desenvolvimento de protocolos e planejar estratégias de cuidados que possam promover melhoria no atendimento e uma maior qualidade de vida para o paciente, juntamente com a toda a equipe de saúde (Cofen, 2018).

Contudo, não existem potenciais evidências para recomendação destas, portanto devem ser utilizadas e acompanhadas pelos serviços de dermatologia, estomaterapia e/ou infecção hospitalar. Dentre os cuidados, citam-se a higiene da pele com produtos adequados (sabonetes com pH ácido), aplicação de selante ou protetor de pele (creme barreira), não uso de produtos oleosos, uso de curativos profiláticos finos (quando clinicamente necessário) para evitar lesões por pressão ou proteger áreas lesadas, remoção da máscara do rosto por 15 minutos, a cada duas horas, fora das áreas de contato do paciente. Se esse período de tempo não for prático, deve-se levantar a máscara pelos lados por 5 minutos, a cada duas horas. Tal informação foi corroborada nos artigos pesquisados, no qual alerta que os cuidados com a pele começam desde o momento do banho, assim como a hidratação deve ocorrer pelo menos uma vez ao dia, de preferência após a higienização diária (Coelho et al., 2020).

O posicionamento em PRONA no paciente tem como principal complicação as lesões por pressão (LPP). O efeito fisiológico da posição PRONA é a melhora da oxigenação em 70\% a 80\% em relação aos valores basais dos pacientes com SDRA. Os mecanismos relacionados à respiração estão associados ao posicionamento do paciente no leito e de acordo com este, pode reduzir o risco de atelectasias e aperfeiçoar a redistribuição da ventilação e perfusão alveolares (Coelho et al., 2020).

A categoria 2 mostra que o tempo de internação prolongado em UTI é considerado em muitos estudos como um dos fatores de risco para LPP, um tempo maior que 10 dias em UTI é um fator fortemente associado a LPP. Em contrapartida, Otto 
et al., (2019) obteve em seu estudo que pacientes apresentaram a integridade cutânea comprometida, em uma média de 2,7 dias de internação, tempo inferior ao encontrado em nosso estudo, que foi de 14,51 dias. Essa diferença pode estar relacionada a adição de outros fatores predisponentes ao surgimento de LPP, como idade, condição nutricional, patologias associadas e medidas protetivas de alívio da pressão (Ramalho et al., 2020).

Quanto à localização das LPP evidenciadas através dos estudos, os autores constataram que os principais locais de desenvolvimento de LPP foram a região sacral e calcâneos, consideradas locais de apoio quando o paciente está em decúbito dorsal ou lateral (comuns entre pacientes críticos). Em relação às categorias, os estágios I e II são as mais prevalentes, confirmando nos resultados obtidos dos estudos e dados da literatura atual (Otto et al., 2019).

Observa-se na categoria 3 que outro fator que deve ser avaliado é a nutrição do paciente. Indivíduos com déficit nutricional ou desidratação, em suma apresentam perda de massa muscular e de peso, tornando os ossos mais salientes. Como sinal de déficits nutricionais têm o edema e a redução do fluxo sanguíneo que ocasiona em lesões isquêmicas. Cabendo ressaltar que pacientes com a nutrição inadequada apresentam risco duplicado de desenvolver LPP (Payne, 2020).

Entretanto conforme mencionado antes, mesmo com todas as recomendações existentes, a prática clínica tem mostrado um número relevante de lesões em áreas de proeminências ósseas, com aspecto isquêmico, similares à LP tissular profundas em pacientes com COVID-19, desenvolvidas durante o uso de ventilação mecânica invasiva, instabilidade hemodinâmica, uso de drogas vasoativas, insuficiência múltipla de órgãos, entre outros fatores (Ramalho et al., 2021).

\section{Conclusão}

Na COVID-19 a posição PRONA apresentou bons resultados para o quadro de insuficiência respiratória aguda. Em contrapartida, tal posição pode ocasionar em lesão por pressão tendo em vista a impossibilidade de mudança de decúbito durante o período de PRONA. Visando a prevenção de lesão por pressão em paciente internados com COVID-19 na UTI, o papel da enfermagem consiste em identificar os fatores de riscos, programar medidas de prevenção e realizar a assistência de enfermagem, entretanto deve durante a realização da assistência deve observar a integridade do tecido tegumentar principalmente durante o banho no leito do paciente, em especial os pacientes que apresentam limitações em seus movimentos. Todos os fatores de riscos devem ser considerados importantes, entretanto levando em consideração fatores predispostos do paciente como características como fator imunológico, tempo de internação, aspecto nutricional entre outro.

Além disso, fatores organizacionais também devem ser analisados, tais como recursos materiais e humanos disponíveis, logística adequada para realização das estratégias de prevenção, entre outros. A literatura disponível ainda não traz evidências científicas que possam associar diretamente a infecção por COVID-19 e o desenvolvimento de lesões de pele decorrentes ou não de pressão.

Portanto é responsabilidade dos profissionais de enfermagem realizar de forma primordial a avaliação dos fatores de riscos para o desencadeamento de LPP em seus pacientes, com a finalidade de prevenir e reconhecer de maneira precoce as LPP e desenvolver parâmetros essenciais para a assistência ligada à integridade da pele do paciente. Sendo assim, o artigo fornece subsídios e base científica para produção de estudos futuros na área, visto que a temática é nova e ainda pouco explorada.

\section{Referências}

Araújo, M. S. et al. (2021). Prone positioning as an emerging tool in the care provided to patients infected with COVID-19: a scoping review. Rev. Latino-Am. Enfermagem. 29, e3397. https://doi.org/10.1590/1518-8345.4732.3397

Alencar, G. S. A., Silva, N. M. \& Assis, E. V. (2018). Lesão por pressão na Unidade de Terapia Intensiva. Revista Nursing, 21(239), 2124-2128.

Associação Brasileira De Normas Técnicas. Informação e documentação - citações em documentos - apresentação: Nbr 10520. Rio de Janeiro, 2002. 
Bardin, L. (2012). Análise de conteúdo. São Paulo: Edições 70.

Brasil. (2020). Ministério da Saúde (BR). Secretaria de Atenção especializada à saúde. Departamento de Atenção Hospitalar, domiciliar e de urgência. Protocolo de manejo clínico da COVID-19 na atenção especializada [Internet]. Brasília: Ministério da Saúde.

Brito, K. K. G., Soares, M. J. G. O. \& Silva, M. A. (2014). Cuidado de enfermagem nas ações preventivas nas úlceras de pressão. Revista Brasileira de Ciências da Saúde, 12(40), 56-61.

Coelho, M. M. F. et al. (2020). Lesão por pressão relacionada ao uso de equipamentos de proteção individual na pandemia da COVID-19. Rev Bras Enferm. 73, e20200670. https://doi.org/10.1590/0034-7167-2020-0670

Conselho Federal De Enfermagem. (2015) Resolução COFEN 0501/2015: regulamentação a competência da equipe de enfermagem as feridas.

Cofen. Covid-19: Orientações sobre a colocação e retirada dos equipamentos de proteção individual (EPIs). In: Enfermagem CFd, editor. 2020.18.

Cofen. Conselho Federal de Enfermagem. Resolução no 567 de 2018. Regulamenta a atuação da equipe de enfermagem no cuidado aos pacientes com feridas. Brasília (DF): COFEN.

Debon, R. et al. (2018). M. A visão de enfermeiros quanto a aplicação da escala de Braden no paciente idoso. Revista de Pesquisa: Cuidado é Fundamental. $10(3), 817-23$.

Duim, E. et al. (2015). Prevalência e característica das feridas em pessoas idosas residentes na comunidade. Rev Esc Enferm USP, $49(5), 51-57$.

Favreto, F. J. L. et al. (2017). A. O papel do enfermeiro na prevenção, avaliação e tratamento das lesões por pressão. Revista gestão \& Saúde. $17,37-47$.

Frazão, J. M., Reis, M. N. de S. dos. \& Silva, S. L. da. (2019). Abordagem do enfermeiro na prevenção de feridas em pacientes hospitalizados. Revista Enfermagem Atual, 88(26), 1-9.

Frederico, G. A. et al. (2018). Integralidade no cuidado de enfermagem as pessoas com ulceras cutâneas. Revista de Enfermagem UFPE on line. 12(7), 19972011.

Fonseca, R. L. et al. (2018). A humanização no cuidado de enfermagem aos pacientes com feridas. Rev.digital, 171. Recuperado de: https://www.efdeportes.com/efd171/a-humanizacao-no-cuidado-de-enfermagem.htm

Geovanini, T. (2016). Tratado de feridas e curativos: enfoque multiprofissional. SP: Rideel.

Gomes, R. K. G. et al. (2018). Prevenção de lesão por pressão: segurança do paciente na assistência à saúde pela equipe de enfermagem. Revista Expressão Católica Saúde, 3(1). DOI: 10.25191/recs.v3i1.2164

Kim, R. S. \& Mullins, K. (2016). Preventing facial pressure ulcers in Acute Respiratory Distress Syndrome (ARDS). Journal of Wound, Ostomy and Continence Nursing, 43(4), 427-429.

Lamão, L. C. L., Quintao, V. A. \& Nunes, C. R. (2016). Cuidados de enfermagem na prevenção de lesão por pressão. Revista Científica Interdisciplinar. 1(1). Recuperado de http://www.multiplosacessos.com/multaccess/index.php/multaccess/article/view/10.

Mascarenhas, V. H. A. et al. (2020). COVID-19 and the production of knowledge regarding recommendations during pregnancy: a scoping review. Rev. Latino-Am. Enfermagem. 28, e3348. https://doi.org/10.1590/1518-8345.4523.3348

Machado, L. C. L. R. et al. (2019). Fatores de risco e prevenção de lesão por pressão: aplicabilidade da Escala de Braden. Revista Eletrônica Acervo Saúde, (21), e635. https://doi.org/10.25248/reas.e635.2019.

Moher, D., et al. (2009). Itens de relatórios preferenciais para revisões sistemáticas e meta-análises: A declaração PRISMA. PLos Medicina. 6 (7).

Moraes, J. T. et al. (2016). Conceito e classificação de lesão por pressão: atualização do national Pressure ulcer advisory panel. Rev. enferm. Cent.-Oeste Min. 6(2), 2292-2306.

National Pressure Ulcer Advisory Panel (NPUAP). (2016). Announces a change in terminology from pressure ulcer to pressure injury and updates the stages of pressure injury [Internet]. Washington: NPUAP.

Nascimento, D. C. et al. (2017). Registro de lesão por pressão: o que é abordado? Revista Hospital Universitário Pedro Ernesto, $15(4), 343-348$.

Otto, C. et al. (2019). Fatores De Risco Para O Desenvolvimento De Lesão Por Pressão Em Pacientes Críticos. Enfermagem em Foco, 10(1), 7-11.

Oliveira, V. M. et al. (2017). Safe prone checklist: construction and implementation of a tool for performing the prone maneuver. Rev Bras Ter Intensiva. 29(2), 131-41.

Pachá, H. H. P. et al. (2018). Pressure Ulcer in Intensive Care Units: a case-control study. Rev Bras Enferm [Internet]. 71(6), 3027-34.

Payne, D. (2020). Skin integrity in older adults: pressure-prone, inaccessible areas of the body. British Journal of Community Nursing, 25(1), 22-26.

Ramalho, A. O. et al. (2020). Reflexões sobre as recomendações para prevenção de lesões por pressão durante a pandemia de COVID-19. ESTIMA, Braz. J. Enterostomal Ther., 18, e2520. https://doi.org/10.30886/estima.v18.940_PT

Ramalho, A. O. et al. (2021). Acute skin failure e lesão por pressão no paciente com Covid-19: um relato de caso. ESTIMA, Braz. J. Enterostomal Ther., 19, e0521. https://doi.org/10.30886/estima.v19.1007_PT 
Research, Society and Development, v. 10, n. 17, e10101724111, 2021

(CC BY 4.0) | ISSN 2525-3409 | DOI: http://dx.doi.org/10.33448/rsd-v10i17.24111

Silva, A, Pierot, E. V \& Vasconcelos, C. D. A. (2020). Lesão por pressão no contexto da pandemia covid-19. In: Congresso Internacional de Produção Científica em Enfermagem. ENFservic.1(1), 60.

Sobest - Associação Brasileira de Estomaterapia. (2017). Associação Brasileira de Enfermagem em Dermatologia (SOBEND). Classificação das lesões por pressão concenso NPUAP 2017 - Adaptada culturamente para o Brasil.

Sousa, C. J., et al. (2020). Aplicação da escala de Braden como fator preventivo de lesão por pressão em unidade de terapia intensiva, Rev., Curitiba, 4(4), 2336-2354.

Souza, M. F. C., et al. (2018). Risco de lesão por pressão em UTI: adaptação transcultural e confiabilidade da EVARUCI. Acta paul. Enferm., 31(2), 201-208.

Soares, C. B., et al. (2014). Revisão Integrativa versus Revisão Sistemática. Reme: Revista Mineira de Enfermagem, 12(4), 758-764.

Teixeira, E., et al. (2013). Integrative literature review step-by-step \& convergences with other methods of review. Rev Enferm UFPI, Teresina, 2, 3-7.

Tolfo, G. R., et al. (2020). Atuação do enfermeiro no cuidado de feridas crônicas na Atenção Primária à Saúde: revisão integrativa. Journal of Chemical Information and Modeling, 53(9), 1689-1699.

Ursi, E. S. (2005). Prevenção de lesões de pele no perioperatório: revisão integrativa da literatura. Dissertação de mestrado, Programa de enfermagem fundamental - Universidade de São Paulo. 1-130.

Urquiza, M. D. A. \& Marques, D. B. (2016). Análise de conteúdo em termos de Bardin aplicada à comunicação corporativa sob o signo de uma abordagem teórico-empírica. EntreTextos, 16(1), 115-144.

Vargas, R. G. \& Santos, L. P. (2019). Prevenção de lesão por pressão em UTI - aplicabilidade da escala de Braden. Revista Pró- UniverSUS, 10(1), 162-165.

Vasconcelos, J. M. B. \& Caliri, M. H. L. (2017). Ações de enfermagem antes e após um protocolo de prevenção de lesões por pressão em terapia intensiva. Esc Anna Nery, 21(1), e20170001. 